\title{
Lunar Ranging, Gravitomagnetism, and APOLLO
}

\author{
T.W. Murphy Jr.
}

Received: 19 January 2009 / Accepted: 26 January 2009 / Published online: 20 February 2009

(C) The Author(s) 2009. This article is published with open access at Springerlink.com 2009

\begin{abstract}
The technique of lunar laser ranging (LLR) has for many decades contributed to cutting-edge tests of the fundamental nature of gravity. These include the best tests to date of the strong equivalence principle, the time-rate-of-change of the gravitational constant, gravitomagnetism, the inverse square law, and preferred frame effects. The phenomenologies of each are briefly discussed, followed by an extended discussion of gravitomagnetism. Finally, the new APOLLO project is summarized, which achieves range precision as low as one millimeter.
\end{abstract}

Keywords Gravitomagnetism · Lunar laser ranging

\section{Introduction}

Lunar laser ranging (LLR) is a technique in which short pulses of laser light are sent from the earth to the moon, reflecting off of arrays of corner cube prisms placed on the moon's surface by astronauts or unmanned missions (Bender et al. 1973; Dickey et al. 1994). The round-trip time is accurately measured, from which the earth-moon distance may be deduced. Comparison to a sophisticated model containing not only gravitational dynamics of the solar system, but also body torque effects, earth tides, surface loading effects, atmospheric propagation delay, etc. allows one to test whether general relativity can adequately describe the lunar orbit, and parameterize any necessary correction.

Because the "Nature of Gravity" conference focused much attention on the gravitomagnetic phenomenon, this paper is largely devoted to that topic, with comparatively little treatment of LLR science in general or the APOLLO project. External references provide ample coverage of these topics.

T.W. Murphy Jr. ( $₫)$

University of California, San Diego, 9500 Gilman Dr., La Jolla, CA 92093-0424, USA

e-mail: tmurphy@physics.ucsd.edu 


\section{LLR Science}

A continuous record of laser range measurements between Earth and Moon dating back to 1969 have provided an unprecedented set of data by which to understand dynamics within the solar system and test the fundamental nature of gravity. A model containing all anticipated physical processes impacting the measurement is constructed to simulate the measurements (see Williams et al. 1996 and references within). Differences are minimized by parameter adjustment, many of which represent initial conditions for the numerical integration of solar system bodies. Because each planet and each effect has a unique signature in frequency space-via harmonic distortions on the lunar orbit-a long time span of data allows one to separate effects and isolate influences. Part of the model describes gravity, which is generally formulated in a parameterized post-Newtonian (PPN) framework, described in Will and Nordtvedt (1972). Fits may be configured to test specific violations of general relativity by freezing or releasing various parameters in any combination. Thus far, no departures from Einstein's prescription for gravity have been identified. Such tests include:

- Weak Equivalence principle to $\Delta a / a \approx 1.3 \times 10^{-13}$ (Williams et al. 2004)

- Strong Equivalence Principle to $\eta<4.5 \times 10^{-4}$ (Williams et al. 2004)

- Evolution of the gravitational constant to $\dot{G} / G<9 \times 10^{-13}$ (Williams et al. 2004)

- Gravitomagnetism to $\approx 0.1 \%$ (Murphy et al. 2007; Soffel et al. 2008)

- Geodetic precession to $<0.6 \%$ (Williams et al. 2004)

- Inverse square law good to $<10^{-10}$ times strength of gravity at $\sim 10^{8} \mathrm{~m}$ scales.

The phenomenologies related to each of the above measurements vary. For instance, the equivalence principle violations (weak or strong) manifest as a displacement, or polarization of the lunar orbit, showing up as a $\cos D$ perturbation, where $D$ is the lunar phase angle with respect to the Earth-Sun line (Nordtvedt 1968). A change in $G$ looks like an anomalous evolution of the phase of the large $\left(2 \times 10^{7} \mathrm{~m}\right)$ elliptical signature of the lunar orbit, in which case the phase anomaly evolves quadratically for a constant $\dot{G}$. Gravitomagnetism imposes both $\cos D$ and $\cos 2 D$ perturbations on the orbit (further discussion below). An anomalous precession rate could be interpreted multiple ways, and so is less clear than other perturbations at singling out a specific failure in the theory. For instance, anomalous

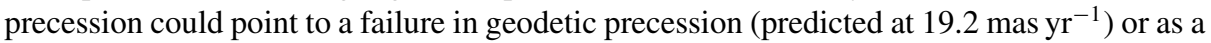
"fifth force" modifying the inverse square law_potentially connected to brane-world ideas of Dvali et al. (2003) and Lue and Starkman (2003). LLR can also provide tests of preferred frames (Müller et al. 1996; Nordtvedt 1987) and of Newton's third law (Nordtvedt 2001).

In general, the lunar orbit provides probes to a wide variety of gravitational phenomena, with a rich set of (mostly) unique "fingerprints" by which to distinguish among them. We now turn attention to the topic of gravitomagnetism, and the role it plays in LLR.

\section{Gravitomagnetism}

\subsection{Introduction to Gravitomagnetism}

A covariant theory of gravity must allow the transformation from one coordinate frame to another while maintaining the ability to describe the same phenomenology in a consistent manner. Such transformations in general produce non-zero space-time components in the metric $\left(g_{0 i} \neq 0\right)$, introducing frame-dependent terms in the equations of motion. Such 
effects are termed gravitomagnetic, in analogy to the magnetic field, which plays a similar role in producing a covariant framework for electromagnetism. Schutz (2003) gives a well-constructed phenomenological derivation of the need for gravitomagnetism in order to satisfy frame independence, for a system of two oppositely directed linear mass currents. Gravitomagnetic phenomena are not readily apparent in human experience due to a $v^{2} / c^{2}$ suppression factor, amounting to $\sim 10^{-8}$ times the gravito-electric (Newtonian) influence in the case of earth's orbit about the sun. Precision measurements within the solar system, however, are sensitive to such small effects.

For a point mass, or for a mass element within a system of masses, one may always transform into the frame of the mass and eliminate the gravitomagnetic field generated by that mass-much as one may move along with an electric charge to "kill" any magnetic field, leaving only the electric field. As soon as two masses are in motion with two different velocities, it is impossible to find a non-rotating frame in which the gravitomagnetic field is zero. Such is the case for rotating bodies, where the mass currents of the individual mass elements combine to produce a net gravitomagnetic field that cannot be transformed away by rectilinear boosts - though it could be eliminated in a frame rotating with the object, incurring many practical difficulties in formulating the external universe in such a frame.

\subsection{Familiar Examples}

Well-known examples of gravitomagnetic phenomenology are the Lense-Thirring precession and the Schiff precession, both involving rotating massive bodies. In the first case, the inclined orbital plane of a satellite moving around the rotating mass will be seen to rotate with respect to the fixed stars. In the latter case, a gyroscope will precess due to the massive body's rotation, the direction and magnitude depending on the latitude of the gyroscope relative to the rotation axis. Both phenomena are commonly called "frame-dragging," and are effects sought by the LAGEOS and the Gravity Probe-B experiments, with some success (these may ultimately achieve $\sim 5 \%$ and $\sim 10 \%$-level confirmation, respectively).

It is tempting to refer to these phenomena as spin-orbit and spin-spin coupling, respectively, in analogy with quantum mechanics. But this is potentially misleading, since massive bodies do not possess intrinsic gravitational "spin," but rather simply have a net angular momentum due to the superposition of many mass elements in instantaneous rectilinear motion, each contributing infinitesimally and coherently to the total angular momentum. We will now look at the gravitomagnetic influence from individual masses, whichwhen superimposed - constitute the familiar composite gravitomagnetic field from a rotating body.

\subsection{Generalized Gravitomagnetism and Application to the Moon's Orbit}

The Earth-Moon system also exhibits a gravitomagnetic dependence, in a form that one might be tempted to call orbit-orbit coupling in the misleading jargon of the previous paragraph. In this case, the earth moving around the sun produces gravitomagnetic field (when assessed in the solar system barycenter frame-or any frame not moving with the earth, for that matter). It is important to note that this gravitomagnetic field does not arise from rotation, but rather from rectilinear motion. The moon moves through this field, experiencing a Lorentz-like force perpendicular to both its velocity and the gravitomagnetic field. The gravitomagnetic acceleration is given by:

$$
\mathbf{a}_{\mathrm{GM}}=\frac{2\left(1+\gamma+\alpha_{1} / 4\right)}{c^{2}} \mathbf{v}_{m} \times \mathbf{v}_{e} \times \mathbf{g}_{m e}
$$


where subscripts $e$ and $m$ denote earth and moon, $\mathbf{v}$ is a velocity vector, and $\mathbf{g}$ is the familiar gravito-electric acceleration vector acting on the moon from the earth. The pre-factor evaluates to 4 in general relativity, with PPN parameters $\gamma=1$, and $\alpha_{1}=0$. The gravitomagnetic field is then $\mathbf{B}=4\left(\mathbf{v}_{e} \times \mathbf{g}_{m e}\right) / c^{2}$, so that the Lorentz acceleration has the familiar $\mathbf{v} \times \mathbf{B}$ form. In Murphy et al. (2007), it is shown that this term in the equations of motion, when integrated over individual mass elements in rotating bodies and applied to the geometry of a

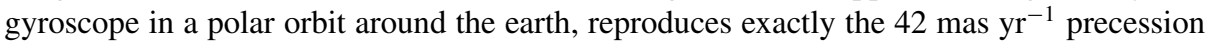
rate sought by GP-B. It also produces $\sim 6 \mathrm{~m}$ amplitude terms in the moon's orbit as evaluated in the solar system barycenter (SSB) frame, in both $\cos D$ and $\cos 2 D$ functions, where $D$ is the phase angle of the moon ( $D=0$ is new moon, $D=180^{\circ}$ is full moon). Having determined these amplitudes in fitting LLR data to sub-centimeter accuracy, we may conclude that gravitomagnetism has been checked in the solar system to $\sim 0.1 \%$ precision using LLR.

\subsection{More than a Coordinate Effect?}

A natural objection to this statement, as articulated by Kopeikin (2007), is that the gravitomagnetic phenomenology in the lunar orbit is dependent on the frame of evaluationevaluating in a geocentric frame would null the rectilinear gravitomagnetic influence of earth on the moon. The implication is that, in transforming to the SSB frame, one inserts phenomenological corrections that are trivially recovered in the fit to the data. Such a procedure would indeed be vacuous, and would do nothing to corroborate the existence of gravitomagnetism. But aside from the practical difficulties of formulating solar system dynamics in a geocentric frame, we must understand whether the choice to execute a coordinate transformation indeed renders the gravitomagnetic sensitivity of LLR meaningless. Note that if the LAGEOS or GP-B were evaluated in a frame rotating with the earth, the "frame dragging" from the rotating earth would disappear, replaced by a host of rotating frame "effects." But does such a coordinate system choice also deprive these precession phenomena of value, if measured? To illuminate this issue, we must detail the manner in which the lunar range model is formulated. In doing so, we will expose the notion that the gravitomagnetic phenomenon is intimately connected with time transformation.

\subsection{Inside LLR Analysis}

Consider the fundamental measurement constituted by lunar ranging. A clock on earth, measuring proper time at some defined gravitational potential, is used to timestamp both outgoing pulses and incoming pulses from the moon. The time at the moon bounce is never measured-nor is an actual distance measured. Two times constitute the measurement, each with an absolute uncertainty of about $10^{-8} \mathrm{~s}\left(0.3 \mathrm{~mm}\right.$ at Earth's velocity of $\left.30 \mathrm{~km} \mathrm{~s}^{-1}\right)$, and a differential uncertainty of about $10^{-11} \mathrm{~s}$ (3 mm at the speed of light). Thus each "range" measurement fundamentally consists of two time measurements on the clock. To transform to the SSB frame, the earth-clock proper times, $\tau$, are converted to SSB frame coordinate times, $t$, according to the prescription formulated by Moyer (1981). In its most basic form:

$$
d t \approx\left(1-\Delta \phi+\frac{1}{2} \dot{s}^{2}\right) d \tau,
$$

where $\Delta \phi$ represents the potential difference between the two locations, and $\dot{s}$ represents the velocity of the clock with respect to the SSB frame. Thus the complete time transformation amounts to gravitational redshift and time dilation corrections. The velocity of the earth in the SSB frame is modulated at the $\cos D$ frequency due to its orbit around the Earth-Moon 
barycenter. But this amounts to only $12 \mathrm{~m} \mathrm{~s}^{-1}$, imposing only a $1.5 \mathrm{~mm} \cos D$ modulation on top of the roughly constant $1.9 \mathrm{~m} \dot{s}^{2}$ correction, modulated annually by $\sim 60 \mathrm{~mm}$ due to earth's eccentric orbit. Following the time transformation, a light propagation correction is applied to account for the fact that the propagation path transits a varying gravitational potential. Called the Shapiro delay, it is computed by the formula:

$$
\Delta t=\frac{(1+\gamma) G M_{s}}{c^{3}} \ln \left(\frac{R_{e}+R_{m}+R_{e m}}{R_{e}+R_{m}-R_{e m}}\right),
$$

where the $R_{x}$ values are the radial coordinates of the bodies from the sun, and $R_{e m}$ is the Earth-Moon distance. This term amounts to a nearly constant $25 \mathrm{~ns}(7.5 \mathrm{~m})$ correction, modulated by only $\sim 10 \mathrm{~mm}$ at the $\cos D$ frequency $(29.53 \mathrm{~d}), \sim 0.8 \mathrm{~m}$ at the anomalistic month frequency $(27.55 \mathrm{~d})$, and $\sim 0.25 \mathrm{~m}$ at an annual frequency. Finally, the earth body figure is Lorentz contracted ( $31 \mathrm{~mm}$ effect) along its velocity vector. To summarize these steps:

1. Obtain launch and return times on a clock at the surface of the Earth;

2. Transform these times to the SSB frame according to the potential energy and velocity of the clock;

3. Compute the Shapiro propagation correction for consideration in the fit;

4. Lorentz contract the earth by a few centimeters;

5. Attempt to fit the observations with a physical model resting on the relativistic equations of motion.

For the last step, with the SSB-corrected times and propagation-correction in hand, one asks the question: are there any world lines for the solar system bodies (Earth and Moon foremost) following the dynamics set out by the equations of motion that allow the time measurements to be consistent with the model? In other words, can a fit be performed that is consistent with general relativity, or PPN modifications thereof? Note that orbits are never constructed in one frame and transformed into another frame: orbits are only constructed once, during the world-line fit to the transformed time coordinates.

As mentioned before, the gravitomagnetic term in the equations of motion produces $\sim 6 \mathrm{~m}$ amplitude effects at $\cos D$ and $\cos 2 D$. The transformation steps detailed above do not insert these phenomenological signatures directly, so the claim that the LLR sensitivity to gravitomagnetism is trivially constructed is not borne out by this analysis.

\subsection{Experimental Conflict Scenario}

The crux of the mater is: if some other experiment claimed a departure of gravitomagnetism from general relativity at, say, the $1 \%$ level, we would be left asking the question: what part of the LLR analysis did we not understand? And fundamentally, it comes down to basic time transformation. This is another way to understand the statement that gravitomagnetism is fundamentally tied to transformations. Gravitomagnetism is the piece that lets gravity be put on a covariant footing, via coordinate transformations. While arbitrarily complex mass currents may mask this fact due to emergent (e.g., "spin") phenomenology, the only reason that gravitomagnetism exists at all is to permit frame transformation (see Schutz 2003). Some have tried to split gravitomagnetism into "intrinsic" and "gauge" forms, but there is no physical basis for this distinction. We do not do the same for magnetic fields in electromagnetism, despite identical frame-dependence features. Similarly, we do not say that the Coriolis "force" is fundamentally different when manifested in a swirling, draining 
tub (spin system) than when seen in the deflection of a projectile (orbit system), despite seemingly disparate phenomenologies.

Perhaps a fair way to put it is: the mere fact that we can successfully fit the lunar orbit in the SSB frame is striking confirmation that gravitomagnetism plays its expected role. One may wish to call the LLR connection to gravitomagnetism "gauge-dependent," and this is fair enough. But such is gravitomagnetism as a whole. The fact that a frame exists in which the gravitomagnetic field from the moving earth is zero does not diminish the physical reality of that field in shaping orbits in the SSB frame.

If another experiment were to claim a $1 \%$ violation of gravitomagnetic phenomenology, the LLR result must be confronted. How can we modify the strength of this term-this physics - to suit one experiment without irreparably damaging the LLR fit? One might investigate which other terms in the equations of motion might also be modified to cover the sins of the delinquent gravitomagnetic term. But each term brings its own unique phenomenological signature. In the case of gravitomagnetism, it is $-6.1 \mathrm{~m}$ in $\cos D$ and $-6.5 \mathrm{~m}$ in $\cos 2 D$. Other terms will not be able to mask this without unraveling some other periodic signatures. Soffel et al. (2008) recently explored these possibilities with a full model fit to LLR data, and found that indeed one has no freedom to change the scaling of the gravitomagnetic term in an ad-hoc (the most versatile) way by more than $0.15 \%$ without destroying the fit to LLR data — even when other terms were permitted to take up the slack.

But modifying the gravitomagnetic strength in isolation-or arbitrarily in relation to the strengths of other terms - in this ad-hoc way lacks physical justification. A more meaningful test is to stay within the framework of the PPN formalism, which amounts to changing $\gamma$ or $\alpha_{1}$ to influence the strength of the term in (1). In this case, other terms in the equations of motion change in a way that preserves covariance automatically, with the caveat that $\alpha_{1}$ does describe a preferred frame in space. Here, Soffel et al. (2008) found that LLR provides a similarly restrictive limit on alteration of the gravitomagnetic term, at the $0.2 \%$ level.

\subsection{Summary Statement}

The point from this discussion is that the LLR sensitivity to gravitomagnetism represents a real limit in physics. It cannot be dismissed as merely an artifact of frame transformation: a "signal" injected and recovered. There are no $\cos D$ or $\cos 2 D$ terms inserted in the transformations of (2) or (3) at the six meter level (few millimeter level, yes). In fact, one can say that gravitomagnetism is intimately connected to transformation properties. Like the magnetic field in electromagnetism, it serves to provide a covariant basis for gravity. We know we can transform magnetic fields away with boosts, but this does not lead us to conclude that such magnetic fields are any less "real" when measured by a compass needle. Likewise, the gravitomagnetic influence on the lunar orbit is real and physical in the SSB. The fact that the LLR clock acts consistently with gravitomagnetism while moving through the SSB frame only serves to highlight the fundamental connection: gravitomagnetism is by its very nature a frame-dependent piece of physics. Interesting arrangements of mass currents do not change that simple fact.

\section{APOLLO}

For the last decade or more, LLR data quality has settled to the impressive level of $\sim 2 \mathrm{~cm}$ range uncertainty. Though the range capability has been roughly constant over this time, the science gains represented in Sect. 2 have seen steady improvement over the years as more 
data accumulates. Because LLR has provided such an extensive array of cutting-edge tests of gravity, it is worth pushing the technique further in order to achieve the best tests possible of the fundamental nature of gravity.

The Apache Point Observatory Lunar Laser-ranging Operation (APOLLO: Murphy et al. 2008) was established in pursuit of this goal. By implementing LLR on a large $(3.5 \mathrm{~m})$ astronomical telescope with modern technology, it is possible to greatly exceed the photon rate experienced by other LLR stations, translating to reduced random uncertainty via sheer statistics. APOLLO routinely achieves random uncertainties in the 1-2 $\mathrm{mm}$ range (Battat et al. 2009), and has seen record photon yields surpassing previous records by almost two orders-of-magnitude. It will take more time and effort to establish whether APOLLO has systematic errors under control at the millimeter level, but so far nothing obvious has emerged. Few-millimeter precision LLR measurements will permit order-of-magnitude improvements in the various tests of the fundamental nature of gravity detailed in Sect. 2. For more information on the details of APOLLO's construction and performance, please consult the references in this paragraph and information online (Murphy 2009). APOLLO's normal points are made public via this website.

Acknowledgements I thank Ken Nordtvedt, Sergei Kopeikin, Eric Michelsen, Jürgen Müller, Slava Turyshev, and Cliff Will for meaningful interactions that have contributed to the articulation of gravitomagnetism herein. I also acknowledge the APOLLO collaboration for their substantial role in establishing the APOLLO experiment, and Jim Williams and Dale Boggs for providing model fits to APOLLO data and associated feedback. APOLLO is funded by the National Science Foundation and by NASA.

Open Access This article is distributed under the terms of the Creative Commons Attribution Noncommercial License which permits any noncommercial use, distribution, and reproduction in any medium, provided the original author(s) and source are credited.

\section{References}

J.B.R. Battat, T.W. Murphy, E.G. Adelberger, B. Gillespie, C.D. Hoyle, R.L. McMillan, E.L. Michelsen, K. Nordtvedt, A.E. Orin, C.W. Stubbs, H.E. Swanson, Publ. Astron. Soc. Pac. 121, 29 (2009)

P.L. Bender, D.G. Currie, R.H. Dicke, D.H. Eckhardt, J.E. Faller, W.M. Kaula, J.D. Mullholland, H.H. Plotkin, S.K. Poultney, E.C. Silverberg, D.T. Wilkinson, J.G. Williams, C.O. Alley, Science 182, 229 (1973)

J.O. Dickey, P.L. Bender, J.E. Faller, X.X. Newhall, R.L. Ricklefs, J.G. Ries, P.J. Shelus, C. Veillet, A.L. Whipple, J.R. Wiant, J.G. Williams, C.F. Yoder, Science 265, 482 (1994)

G. Dvali, A. Gruzinov, M. Zaldarriaga, Phys. Rev. D 68, 024012 (2003)

A. Lue, G. Starkman, Phys. Rev. D 67, 064002 (2003)

S.M. Kopeikin, Phys. Rev. Lett. 98, 229001 (2007)

T.D. Moyer, Celest. Mech. 23, 33 (1981)

J. Müller, K. Nordtvedt, D. Vokrouhlicky, Phys. Rev. D 54, R5927 (1996)

T. Murphy, APOLLO Website, http://www.physics.ucsd.edu/ tmurphy/apollo. Accessed 19 January 2009

T.W. Murphy Jr., K. Nordtvedt, S.G. Turyshev, Phys. Rev. Lett. 98, 071102 (2007)

T.W. Murphy, E.G. Adelberger, J.B.R. Battat, L.N. Carey, C.D. Hoyle, P. Leblanc, E.L. Michelsen, K. Nordtvedt, A.E. Orin, J.D. Strasburg, C.W. Stubbs, H.E. Swanson, E. Williams, Publ. Astron. Soc. Pac. 120, 20 (2008)

K. Nordtvedt, Phys. Rev. 169, 1014 (1968)

K. Nordtvedt, Astrophys. J. 320, 871 (1987)

K. Nordtvedt, Class. Quant. Grav. 18, L133 (2001)

B. Schutz, Gravity from the Ground Up (Cambridge University Press, Cambridge, 2003), pp. 245-253

M. Soffel, S. Klioner, J. Müller, L. Biskupek, Phys. Rev. D 78, 024033 (2008)

C.M. Will, K. Nordtvedt, Astrophys. J. 177, 757 (1972)

J.G. Williams, X.X. Newhall, J.O. Dickey, Phys. Rev. D 53, 6730 (1996)

J.G. Williams, S.G. Turyshev, D.H. Boggs, Phys. Rev. Lett. 93, 261101 (2004) 